\title{
Eficiencia térmica de un calentador solar doméstico de agua hecho con botellas plásticas, Chachapoyas - Amazonas, 2014
}

\section{Thermal efficiency of a solar domestic water heater made of plastic bottles, Chachapoyas - Amazonas 2014}

\author{
Jackeline Mendoza Mestanza ${ }^{1}$, Joherrs Miki RituayYoplac ${ }^{2}$, *Miguel Ángel Barrena Gurbillón ${ }^{3}$
}

\section{RESUMEN}

En la presente investigación se evaluó la eficiencia térmica de un calentador solar doméstico hecho con botellas plásticas; en función a la temperatura del agua en el tanque de almacenamiento, la temperatura ambiente y la radiación solar. Se construyó un calentador solar hecho con materiales no convencionales como: botellas de tereftalato de polietileno (PET) de $1,750 \mathrm{~L}$, cajas de tetra pak de $1 \mathrm{~L}$, tubos de CPVC y PVC, y un tanque de almacenamiento de $80 \mathrm{~L}$. La evaluación se realizó en tres estados climáticos: soleado, nublado y soleado-nublado. Los datos fueron recolectados durante el día desde las 8:00 hasta las 16:00, cada 30 min. Los datos de radiación solar, temperatura ambiente y velocidad del viento fueron brindados por la estación meteorológica ubicada a 300 metros aproximadamente del lugar de experimento. Los días soleados tuvieron una radiación de $5,15 \mathrm{kWh} / \mathrm{m}^{2}, 10 \mathrm{~s}$ combinados $3,79 \mathrm{kWh} / \mathrm{m}^{2}$ y los nublados $2,33 \mathrm{kWh} / \mathrm{m}^{2}$. La temperatura en el tanque de almacenamiento alcanzo para 40,33 y $27^{\circ} \mathrm{C}$ para días soleados, combinados y nublados respectivamente. Se determinó la eficiencia por el cociente de la energía térmica ganada y la energía solar recibida; encontrando la mayor eficiencia (41\%) en los días soleados.

Palabras clave: colector solar, energía térmica ganada, energía solar recibida.

\begin{abstract}
In this research, the thermal efficiency of a domestic solar heater made with plastic bottles; depending on the temperature of the water in the storage tank, the ambient temperature and solar radiation was evaluated. A solar heater made of non-conventional materials such as: $1.750 \mathrm{~L}$ polyethylene terephthalate $(\mathrm{PET})$ bottles, $1 \mathrm{~L}$ tetra pak boxes, CPVC and PVC pipes, and a storage tank of $80 \mathrm{~L}$. The evaluation was performed in three climatic states: sunny, cloudy and sunny-cloudy. The data was collected during the day from 8:00 am to 4:00 pm, every 30 minutes. The solar radiation, ambient temperature and wind speed data, were provided by the meteorological station located approximately 300 meters from the experiment site. The sunny days had a radiation of $5.15 \mathrm{kWh} /$ $\mathrm{m} 2$, the combined $3.79 \mathrm{kWh} / \mathrm{m} 2$ and the cloudy $2.33 \mathrm{kWh} / \mathrm{m} 2$. The temperature in the storage tank reached 40,33 and $27^{\circ} \mathrm{C}$ for sunny days, combined and cloudy days, respectively. The efficiency was determined by the ratio of the thermal energy gained and the solar energy received; finding the highest efficiency (41\%) on sunny days.
\end{abstract}

Keywords: solar collector, thermal energy gained, solar energy received.

\footnotetext{
${ }^{1}$ Ingeniera Ambiental. Universidad Nacional Toribio Rodríguez de Mendoza de Amazonas.

${ }^{2}$ Ingeniero Ambiental. Universidad Nacional Toribio Rodríguez de Mendoza de Amazonas

${ }^{3}$ *Ingeniero Químico. Universidad Nacional Toribio Rodríguez de Mendoza de Amazonas. Correo electrónico:

miguel.barrena@untrm.edu.pe
} 


\section{INTRODUCCIÓN}

En la actualidad el agua caliente constituye un consumo energético importante en un hogar, teniendo diversos usos, como la higiene personal y la limpieza del hogar (Agencia Internacional de Energía, 2006). Un calentador solar se usa con el fin de aprovechar la energía solar, la cual es gratis y no es aprovechada adecuadamente, siendo una opción atractiva para sustituir los calentadores tradicionales teniendo en cuenta las bajas temperaturas requeridas $\left(40^{\circ} \mathrm{C}\right.$ a $\left.60^{\circ} \mathrm{C}\right)$, además del ahorro energético; la fuente de calor es la radiación solar, que sustituye los combustibles fósiles o la electricidad (Burbano et al., 2006).

Los colectores solares son dispositivos que se utilizan para colectar, absorber y transferir energía a un fluido (agua o aire), ésta transferencia de energía puede ser utilizada para calentar agua, en sistemas de calefacción o climatización de piscinas (Cárdenas et al., 2006).

La creciente preocupación por lograr un aprovechamiento de las energías renovables, la necesidad de diversificar las fuentes aprovechables de energía y reducir las emisiones de gases que provocan el efecto invernadero, precursor del cambio climático global, ha permitido introducir nuevas perspectivas en las investigaciones por impulsar el aprovechamiento de tecnologías relacionadas con la energía renovable.

Las termas solares hechas a base de materiales de reciclaje han sido empleadas en diferentes lugares a nivel internacional, con resultados satisfactorios. Es necesario destacar que en el caso de la experiencia desarrollada por Alano (2004), el colector solar para la producción de agua caliente fue construido con botellas de plástico y cajas de tetra pack; obteniendo un resultado positivo, con un funcionamiento perfecto y a la vez una manera responsable de deshacerse de los residuos. El funcionamiento fue similar al de los sistemas comerciales concluyendo que puede calentar agua hasta $38^{\circ} \mathrm{C}$ en invierno y hasta más de $50^{\circ} \mathrm{C}$ en verano.

En la Región Amazonas no se ha realizado investigación con calentadores solares hechos con botellas de plástico; pero Vigo y Trigoso (2012), han construido y evaluado una terma solar con materiales existentes en la zona y con colector de tubos de CPVC, obteniendo las siguientes temperaturas para el agua: días soleados de 38 a $44^{\circ} \mathrm{C}$, siendo la eficiencia del colector de $52 \%$; en días combinados de 30 y $37^{\circ} \mathrm{C}$, con eficiencia de $48 \%$; y en días nublados, hasta $25^{\circ} \mathrm{C}$, con una eficiencia de $30 \%$.
El objetivo de la presente investigación fue determinar la eficiencia térmica de un calentador solar de agua hecho con botellas plásticas.

\section{MATERIAL Y MÉTODOS}

La presente investigación se realizó en la Ciudad Universitaria de la Universidad Nacional Toribio Rodríguez de Mendoza de Amazonas, ubicado en la ciudad de Chachapoyas, Región Amazonas; a 2350 msnm y $17^{\circ} \mathrm{C}$ de temperatura ambiente promedio.

Para la construcción del colector solar se recolectó 50 botellas de tereftalato de polietileno (PET) y 50 cajas de tetra pak. Los tubos fueron de PVC para agua caliente (CPVC) de $1 / 2$ " por $1 \mathrm{~m}$ de largo, unidos con pegamento a niples de $6 \mathrm{~cm}$, tes y codos de $1 / 2$ " de CPVC. Para el almacenamiento de agua caliente se utilizó un tanque de polietileno de $80 \mathrm{~L}$ colocado dentro de una caja de madera cuyo interior se revistió con poliestireno expandido a fin de conservar el agua caliente el mayor tiempo posible. El sistema de abastecimiento de agua a la terma solar tenía una válvula para mantener el volumen de trabajo del agua, reponiéndola conforme vaya consumiendo el usuario.

Se registró los datos de la medición directa de las temperaturas del agua en la entrada al colector, dentro del colector, salida del colector, en el tanque de almacenamiento y del ambiente; también, la radiación solar, desde las 08:00 hasta las 16:00 horas, con intervalos de $30 \mathrm{~min}$; durante 30 días, los cuales fueron clasificados de acuerdo a tipos de días; soleados (presencia masiva de sol), combinados (presencia parcial de sol) y nublados (ausencia visible de sol).

Para calcular la eficiencia térmica se utilizó la siguiente fórmula descrita por Valera (2007).

$\eta \mathrm{E}=$ Energía térmica útil / energía solar recibida

\section{Energía térmica ganada: $\mathrm{ET}=\mathrm{m} . \mathrm{c} . \mathrm{T}$}

Donde $\mathrm{m}=$ masa de agua en la terma $=80 \mathrm{~L}=8 \times 10^{4} \mathrm{~g}$; $\mathrm{c}=$ calor específico del agua $=4,18 \mathrm{~J} \quad \mathrm{~g}^{-1}{ }^{\circ} \mathrm{C} ; \mathrm{T}=$ temperatura máxima del agua $(4: 00 \mathrm{pm})$ temperatura del agua en el tanque al inicio (8:00 am) en ${ }^{\circ} \mathrm{C}$.

\section{Energía solar recibida: $\mathrm{ES}=e s . \mathrm{A}$}

Donde es: energía solar recibida, se calculó por el área bajo la curva de radiación solar vs tiempo, mediante el método del área de los rectángulos bajo la curva; $\mathrm{A}=$ área del colector solar en $\mathrm{m}^{2}$. 


\section{RESULTADOS}

\section{Instalación de terma solar}

La terma solar se ubicó en el techo de la Planta Piloto de la Facultad de Ingeniería y Ciencias Agrarias de la UNTRM, en la orientación norte-sur. El lado sur del colector se levantó $30^{\circ}$ y la torre con el tanque de almacenamiento de agua se ubicó en este lado para que su sombra no llegue al colector.

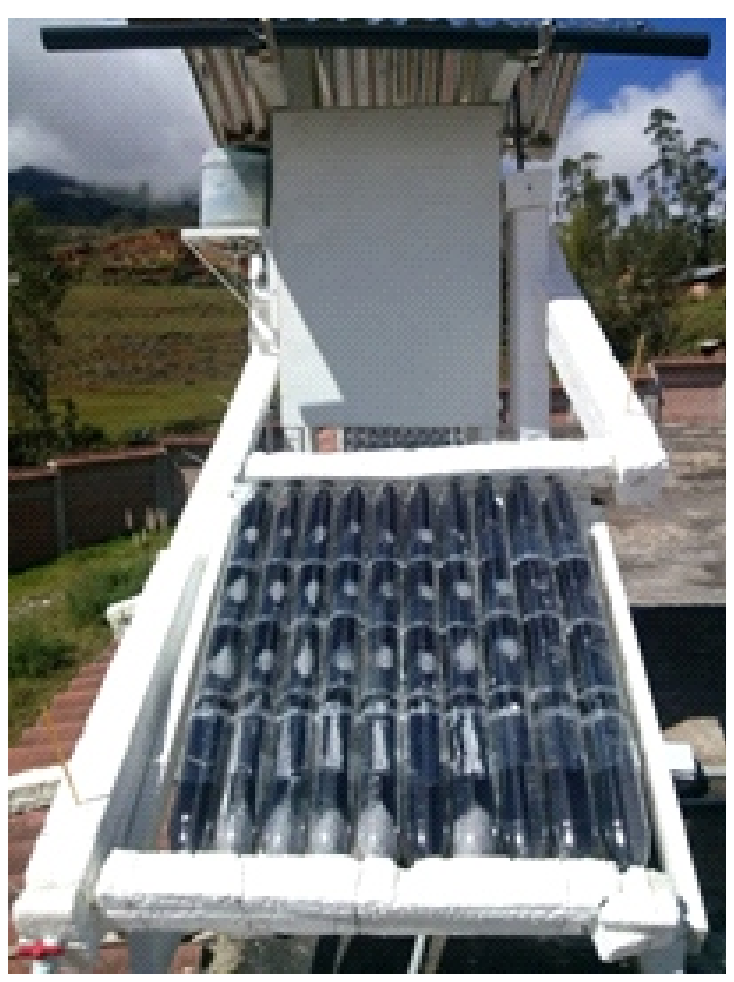

Figura 1. Fotografía de la terma solar instalada para la evaluación

La radiación solar recibida fue de $5,15 \mathrm{kWh} / \mathrm{m}^{-2}$ y la eficiencia $41 \%$ para días soleados (Figura 2), mientras que para días combinados se encontraron valores de $3,79 \mathrm{kWh} / \mathrm{m}^{-2}$ y $39 \%$ en ese mismo orden (Figura 3).

Para los días nublados (Figura 4), los resultados son menores a las dos alternativas anteriores (soleado y combinado) con $2,33 \mathrm{kWh} \mathrm{m}^{-2}$ y $37 \%$ para radiación recibida y eficiencia térmica.

Para cada tipo de día, la ganancia de temperatura del agua en el tanque de almacenamiento y la radiación solar recibida fue: soleados $\left(22,5^{\circ} \mathrm{C} ; 5,15 \mathrm{kWh} / \mathrm{m}^{2}\right)$, combinados $\left(16^{\circ} \mathrm{C} ; 3,79 \mathrm{kWh} / \mathrm{m}^{2}\right)$ y nublados $\left(11^{\circ} \mathrm{C}\right.$; $\left.2,33 \mathrm{kWh} / \mathrm{m}^{2}\right)$.
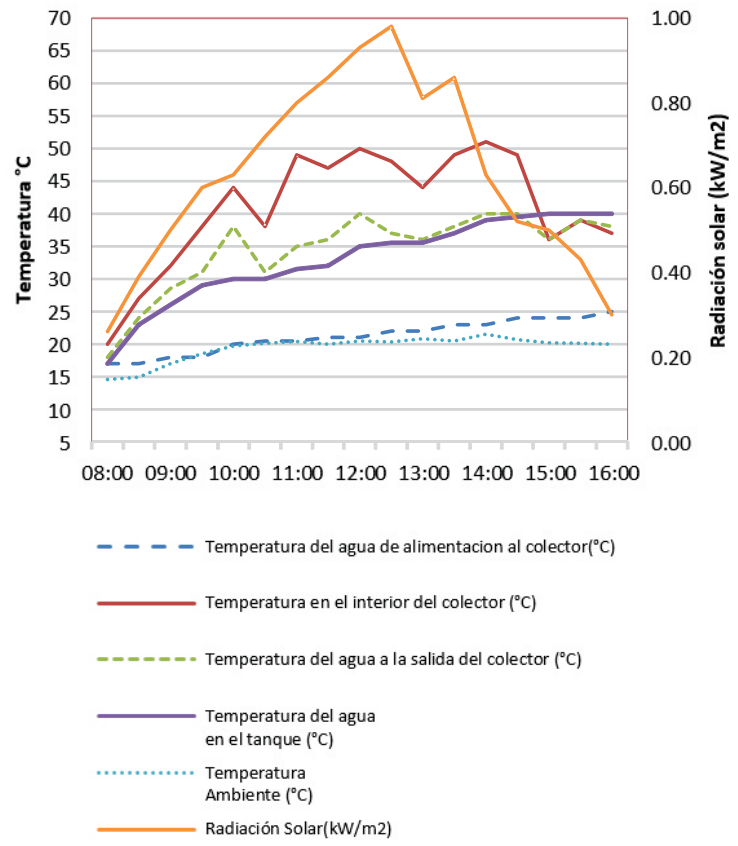

Figura 2. Evolución de la temperatura en los seis puntos de estudio para días soleados

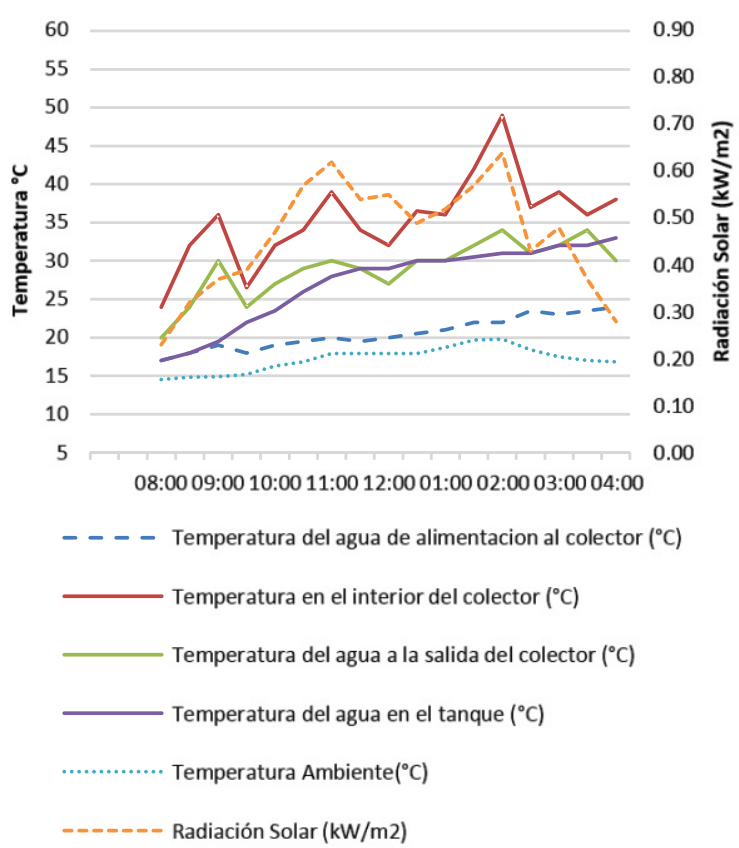

Figura 3. Evolución de la temperatura en los seis puntos de estudio para días soleados 


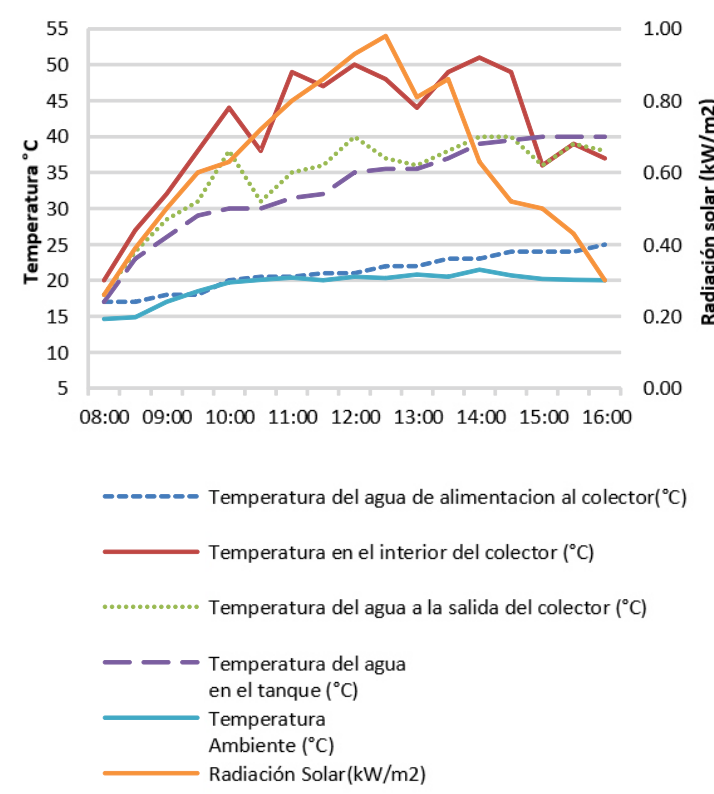

Figura 4. Evolución de la temperatura en los seis puntos de estudio para días soleados

Las temperaturas máximas del ambiente, del colector, del agua alimentada al sistema, del agua a la salida del colector y del agua en el tanque de almacenamiento fue: soleados: $(20,8 ; 51 ; 25 ; 40$; $\left.40^{\circ} \mathrm{C}\right)$, combinados $\left(18,4 ; 24 ; 34 ; 33^{\circ} \mathrm{C}\right)$ y nublados $\left(16,3 ; 19 ; 33 ; 28 ; 27^{\circ} \mathrm{C}\right)$.

\section{DISCUSIÓN}

La tendencia descendente de la eficiencia se debe directamente a la disminución de la presencia visible del sol correspondiente a cada tipo de día en que se hizo la evaluación, tal como se observa en las Figuras 2,3 y 4 .

Las eficiencias obtenidas en días soleados y combinados son menores lo encontrado por Vigo y Trigoso (2012), hasta en 11 y 9\% respectivamente; sin embargo en días nublados, se obtuvo mayor eficiencia hasta $7 \%$ superior; estas podrían deberse a los meses de evaluación.

Los resultados evidencian que se puede aprovechar la energía solar para calentar agua, con una eficiencia térmica entre 37 y $41 \%$, y evitar la emisión de $\mathrm{CO}_{2}$ en hasta 0,75 toneladas al año por $\mathrm{m}^{2}$ de colector solar (Agencia Internacional de Energía, 2006).

\section{CONCLUSIONES}

Un colector solar, en la ciudad de Chachapoyas, puede operar con eficiencias entre 37 y $41 \%$, según la cantidad de la radiación recibida.

El colector solar logra incrementar la temperatura del agua desde $9^{\circ} \mathrm{C}$, la que podría ser empleada para aseo personal.

\section{AGRADECIMIENTO}

Al Instituto de Investigación para el Desarrollo Sustentable de Ceja de Selva de la UNTRM, por brindarnos los datos meteorológicos necesarios para la investigación realizada.

\section{REFERENCIAS BIBLIOGRÁFICAS}

Alano, J. A. (2004). Manual sobre la construcción e instalación del calentador solar con botellas descartables. Tubarao - Santa Catalina.

Agencia Internacional de Energía. (2006). El caso de la tecnología de la terma solar. París.

Burbano, J. C. Restrepo, Á. H., y Sabogal, O. J. (2006). Diseño y construcción de un calentador solar de agua operado por termosifón. Disponible e n http.//micalentadorsolar.com.mx. Acceso el 12/10/14.

Cárdenas, A. E., Quesada, G. y Pérez M. (2006). Incrustaciones en instalaciones solares térmicas. Energía y tú. Revista Científica Trimestral de CUBASOLAR. №36. Pág. 18 -24 .

Valera, A. (2007). Energía solar II. Edición a cargo de la Asamblea Nacional de Recortes. Lima - Perú.

Vigo, L. V.y Trigoso, R. R. (2012). Eficiencia térmica de un calentador solar de agua de 80 litros con colector de $1 \mathrm{~m}^{2}$ (Tesis de grado). Universidad Nacional Toribio Rodríguez de Mendoza de Amazonas. 